\title{
Beta-blockers in septic shock to optimize hemodynamics? We are not sure
}

\author{
Daniel De Backer ${ }^{1 *}$ (iD and Djillali Annane ${ }^{2}$
}

(C) 2016 Springer-Verlag Berlin Heidelberg and ESICM

In recent years, interest in the use of beta-blockade in sepsis has increased, bearing in mind that the septic heart may benefit from some protection against excessive adrenergic stimulation. Then, one trial suggested substantial improvement in survival following heart rate control by continuous infusion of esmolol, a short-acting selective beta-1 antagonist, in a highly selected group of septic shock with severe tachycardia [1]. The same group of researchers suggested potential positive effects on cardiac function from infusion of esmolol in a subsequent study in 45 septic shock with tachycardia above $95 \mathrm{bpm}$ published in this issue [2]. In this study, esmolol-related decrease in heart rate was associated with increased stroke volume (SV). Owing to decreased cardiac contractility (as illustrated by the decrease in $\mathrm{d} P / \mathrm{d} t_{\max }$ ), the preservation of SV can only result from increase in preload (related to the increase in diastolic time) or a decrease in afterload. Yet, the authors ascribed increase in SV to improved ventriculo-arterial coupling, as $\mathrm{MAP} / \mathrm{SV}$, an index of aortic elastance, improved while filling pressures were stable.

However, assessment of ventriculo-arterial coupling was indirect and relied on pulse wave analysis, thermodilution, and basic echocardiography, and most measurements are potentially subject to mathematical coupling of the data. Therefore, further studies using direct and independent assessments of cardiac-arterial coupling are needed before reliable conclusions can be made. Another major limitation was that arterial pressure was measured in peripheral arteries, thus underestimating

\footnotetext{
*Correspondence: ddebacke@ulb.ac.be

${ }^{1}$ Department of Intensive Care, CHIREC Hospitals, Université Libre de Bruxelles, Rue Wayez 35, 1420 Braine l'Alleud, Brussels, Belgium Full author information is available at the end of the article

For contrasting viewpoints, please go to doi:10.1007/s00134-016-4407-3 and doi:10.1007/s00134-016-4414-4.
}

central arterial pressure $[3,4]$. This is due to the impact of reflected waves that are generated at branch points. Accordingly, the amplitude and timing of the central aortic waveform, which directly affects the heart and determines ventriculo-arterial coupling, differ from peripheral arterial traces that are commonly measured in ICU patients (Fig. 1). In addition, as vasodilation decreases the influence of reflected waves (Fig. 1), the discrepancies between central and peripheral waveforms are exacerbated with alterations in vascular tone such as observed in septic shock $[5,6]$. The impact of the reflected waves was nicely illustrated by Bilo et al. [7] who restored a normal arterial trace by compressing the artery distally to the site of measurement, mimicking a restoration of arterial tone. In fluid-resuscitated endotoxemic pigs, Hatib et al. [5] nicely showed that peripheral systolic and mean arterial pressures markedly underestimated central aortic pressures, while diastolic pressure was reliably measured.

Of note, the effects of heart rate manipulation reported by Morelli et al. [2] differed from data observed in middle-aged healthy individuals [8]. Using central measurements of aortic pressure and volumetric catheters to determine aortic volume, Stefanadis et al. [8] observed that pacing did not affect MAP, decreased systolic blood pressure while diastolic pressure was increased, and decreased the augmented pressure (the difference between central and peripheral systolic pressure). Interestingly, distensibility of the aorta increased and stiffness index decreased at high HR, indicating that Ea should decrease at high HR. To what extent the results of Morelli et al. suggesting a decrease in Ea and an improvement in ventriculo-arterial coupling were affected by the site of arterial pressure measurement remains to be determined. Of note, LVEF was unchanged, which suggests that ventriculo-arterial coupling was not improved to the extent suggested by peripheral arterial pressure

\section{Springer}




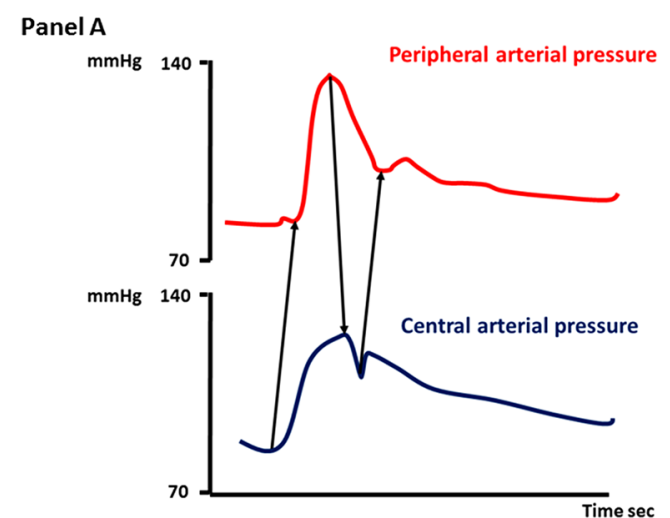

Panel B

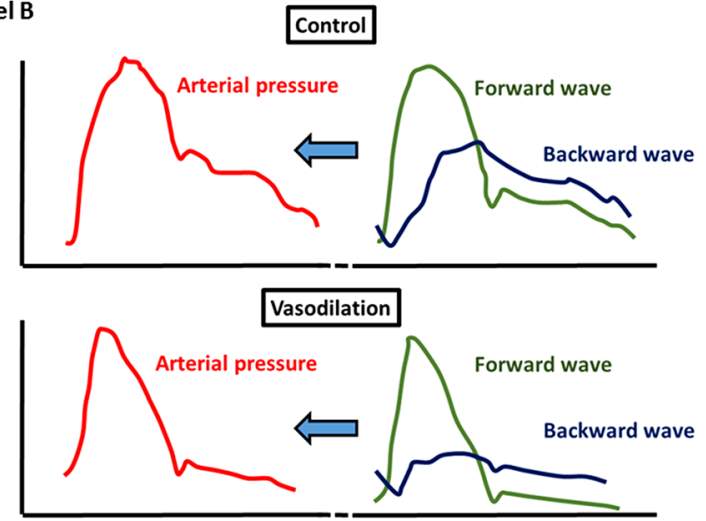

Fig. 1 Influence of reflected waves on arterial traces. a Central and peripheral aortic pressures: under the influence of reflected waves, central and peripheral arterial waveforms differ. Central aortic trace presents an earlier increase in the arterial pressure but the peak systolic pressure is slightly delayed and lower than in the periphery; the dicrotic notch occurs earlier and at a higher pressure than in the periphery (curves redrawn after Wang et al. [4]). b Impact of forward and backward waves on arterial traces: the measured arterial trace reflects a given point of the summation of forward and backward waveforms. Vasodilation alters reflection of waves and mostly decreases the impact of reflected waves. As a result the augmented pressure and dicrotic notch pressures are markedly decreased (adapted from Berger et al. [9])

measurements. Thus, whether esmolol-related heart rate reduction improved ventriculo-(central) arterial decoupling remains uncertain. However, the improvement in peripheral dicrotic notch-diastolic pressure gradient suggests that beta-blockade improved the reflection of waves. It still remains unclear whether this effect resulted from changes in arterial tone or in heart rate.

Finally, optimizing patients' hemodynamics not only improves cardiac efficiency, as evaluated in this study, but also improves tissue perfusion. Unfortunately, the reader is provided with minimal information on the issue of tissue perfusion. As cardiac output decreased by $5 \%$ (a nonstatistically significant drop), oxygen delivery very likely decreased, as observed in the previous trial by the same researchers $(-20 \%$ decrease in oxygen delivery) [1]. Whether the negative impact of the decrease in oxygen delivery may be balanced by potentially beneficial effects on cardiac function remains speculative.

The net findings from the two trials performed by these researchers are that continuous infusion of esmolol may control heart rate without impairing stroke volume in a highly selected group of septic shock with severe tachycardia [1, 2]. Additional investigations are required to determine whether preserved stroke volume results from increased preload despite impaired contractility or from improved ventriculo-arterial coupling following decrease in afterload and Ea.

\section{Author details}

${ }^{1}$ Department of Intensive Care, CHIREC Hospitals, Université Libre de Bruxelles, Rue Wayez 35, 1420 Braine I'Alleud, Brussels, Belgium. ${ }^{2}$ General Intensive Care Unit, Raymond Poincaré Hospital (APHP), Laboratory of Infection and Inflammation, U1173 INSERM and University of Versailles SQY, 104 Boulevard Raymond Poincaré, 92380 Garches, France.

Received: 3 June 2016 Accepted: 9 June 2016

Published online: 27 June 2016

\section{References}

1. Morelli A, Ertmer C, Westphal M, Rehberg S, Kampmeier T, Ligges S et al (2013) Effect of heart rate control with esmolol on hemodynamic and clinical outcomes in patients with septic shock: a randomized clinical trial. JAMA 310:1683-1691

2. Morelli A, Singer M, Ranieri VM, D'Egidio A, Mascia L, Orecchioni A et al (2016) Heart rate reduction with esmolol is associated with improved arterial elastance in patients with septic shock: a prospective observational study. Intensive Care Med. doi:10.1007/s00134-016-4351-2

3. Hebert JL, Lecarpentier Y, Zamani K, Coirault C, Daccache G, Chemla D (1995) Relation between aortic dicrotic notch pressure and mean aortic pressure in adults. Am J Cardiol 76:301-306

4. Wang JJ, O'Brien AB, Shrive NG, Parker KH, Tyberg JV (2003) Time-domain representation of ventricular-arterial coupling as a windkessel and wave system. Am J Physiol Heart Circ Physiol 284:H1358-H1368

5. Hatib F, Jansen JR, Pinsky MR (2011) Peripheral vascular decoupling in porcine endotoxic shock. J Appl Physiol 111:853-860

6. Dorman T, Breslow MJ, Lipsett PA, Rosenberg JM, Balser JR, Almog Y et al (1998) Radial artery pressure monitoring underestimates central arterial pressure during vasopressor therapy in critically ill surgical patients. Crit Care Med 26:1646-1649

7. Bilo HJ, Strack van Schijndel RJ, Schreuder WO, Groeneveld AB, Thijs LG (1989) Decreased reflection coefficient as a possible cause of low blood pressure in severe septicaemia. Intensive Care Med 15:137-139

8. Stefanadis C, Dernellis J, Vavuranakis M, Tsiamis E, Vlachopoulos C, Toutouzas Ket al (1998) Effects of ventricular pacing-induced tachycardia on aortic mechanics in man. Cardiovasc Res 39:506-514

9. Berger DS, Li JK, Laskey WK, Noordergraaf A (1993) Repeated reflection of waves in the systemic arterial system. Am J Physiol 264:H269-H281 